\title{
Um Mecanismo para Potencialização da Agregação de Tráfego em Redes Ópticas Elásticas
}

\author{
Iallen Santos $^{1,3}$, Alexandre Fontinele ${ }^{1}$, Lucas Hiago ${ }^{2}$, José Suruagy Monteiro ${ }^{1}$, \\ André Soares ${ }^{2}$ e Divanilson R. Campelo ${ }^{1}$ \\ ${ }^{1}$ Centro de Informática (CIn) - Universidade Federal de Pernambuco (UFPE) \\ Recife - PE - Brasil \\ ${ }^{2}$ Departamento de Computação - Universidade Federal do Piauí (UFPI) \\ Teresina - PI - Brasil \\ ${ }^{3}$ Instituto Federal de Educação, Ciência e Tecnologia do Piauí (IFPI) \\ São Raimundo Nonato - PI - Brasil \\ igssecin.ufpe.br
}

\begin{abstract}
Elastic optical networks allow for a division of the optical spectrum into frequency slots, which can be combined to create channels with bandwidth defined by demand. Their flexibility provides greater spectral efficiency when compared with traditional optical networks with fixed grids. Traffic grooming strategies allow for even more efficiency in the use of network resources, reducing the number of guard bands and the utilization of transmitters and receivers. This work proposes a mechanism that can be combined with the existing resource allocation solutions to increase the traffic grooming. The experiments show that the adoption of the proposed mechanism also contributes to the reduction of the bandwidth blocking rates without compromising power consumption.
\end{abstract}

Resumo. As redes ópticas elásticas permitem a divisão do espectro óptico em slots de frequência que podem ser combinados para criar canais com largura de banda definida sob demanda. Esta flexibilidade provê maior eficiência espectral em relação às tradicionais redes ópticas com grade fixa. Estratégias de agregação de tráfego permitem atingir uma eficiência ainda maior na utilização dos recursos da rede, reduzindo a quantidade de bandas de guarda e a utilização de transmissores e receptores. Este trabalho propõe um mecanismo que combinado às soluções de alocação de recurso já existentes, potencializa a agregação de tráfego. Os experimentos realizados mostram que a adoção do mecanismo proposto também contribui para a redução das taxas de bloqueio de banda sem comprometer o consumo energético.

\section{Introdução}

A utilização da fibra óptica como meio de transmissão de dados apresenta uma série de benefícios. Entre eles, altas taxas de largura de banda, imunidade a interferências eletromagnéticas e a multiplexação óptica. Tendo isto em vista, as redes ópticas têm potencial para se perpetuar como a melhor alternativa para compor os backbones das redes de transporte. 
A Orthogonal Frequency Division Multiplexing (OFDM) está sendo apontada pela comunidade científica como uma tecnologia promissora para particionar o espectro óptico. A OFDM divide o espectro óptico em porções denominadas slots de espectro que por sua vez podem ser combinadas para formar canais com diferentes amplitudes espectrais.

Os transmissores e receptores que permitem o ajuste da frequência central e a quantidade de slots de espectro utilizados para a transmissão são denominados Bandwidth Variable Transponders (BVTs). Os Bandwidth Variable Cross Conects (BVWXCs) permitem comutar os canais ópticos flexíveis ao longo dos nós intermediários [Wang et al. 2012].

Para estabelecer um circuito óptico entre dois nós em uma rede óptica OFDM, devem ser escolhidos uma rota, um formato de modulação e uma faixa de espectro. Este problema é conhecido como Routing, Modulation Level and Spectrum Allocation(RMLSA) [Christodoulopoulos et al. 2011]. O objetivo das soluções RMLSA é maximizar a capacidade da rede de atender às requisições dos clientes utilizando o mínimo de recursos possível.

O roteamento determina por quais enlaces e nós intermediários será estabelecido um novo circuito. Para cada novo circuito deve ser escolhido um formato de modulação levando em consideração a qualidade do sinal óptico e a eficiência espectral pretendida. Por fim, o algoritmo RMLSA determina quais slots de espectro serão utilizados para alocar cada circuito.

Este trabalho utiliza o termo requisição para se referir a uma requisição feita por um cliente da rede óptica elástica para transmitir um fluxo de dados. Cada requisição consiste em uma tupla $(O, D, B)$ onde $O$ é o nó de origem, $D$ é o nó de destino e $B$ é a largura de banda requisitada. Neste trabalho são abordadas as redes ópticas elásticas com tráfego dinâmico, ou seja, os instantes de chegada das requisições não são conhecidos previamente.

Visando aumentar a eficiência espectral e reduzir a utilização de BVTs, alguns trabalhos da literatura têm proposto estratégias em que diversas requisições são atendidas por um mesmo circuito óptico [Zhang et al. 2015, Costa and Drummond 2016, Wu et al. 2017]. Este tipo de estratégia é referenciada na literatura como agregação de tráfego.

Os BVTs e BV-WXCs permitem a ampliação da capacidade dos circuitos ópticos visando o atendimento de novas requisições através da agregação de tráfego. Entretanto, devido à restrição de contiguidade de espectro, isto pode ser feito apenas quando há disponibilidade de espectro óptico adjacente ao canal óptico em questão [Zhang et al. 2013]. Este trabalho propõe um mecanismo chamado Espaçamento Pró Agregação de Tráfego (EsPAT) que pode ser associado às soluções RMLSA para potencializar a agregação de tráfego em redes ópticas elásticas.

O EsPAT permite a potencialização da agregação de tráfego o que resulta em menores taxas de bloqueio de banda. Nos experimentos realizados obteve-se uma redução de pelo menos $16,7 \%$ e até $90,3 \%$ de bloqueio de banda com a utilização do EsPAT considerando os mesmos algoritmos de agregação de tráfego e RMLSA. Além disso, o mecanismo proposto viabilizou a redução de pelo menos $11,3 \%$ na utilização média de BVTs. 
O restante deste trabalho está organizado da seguinte forma: A Seção 2 apresenta os trabalhos relacionados. A Seção 3 aborda os conceitos de agregação de tráfego e RMLSA. A Seção 4 apresenta os modelos de camada física e de consumo energético, bem como os modelos de BVTs e BV-WXCs considerados nos experimentos realizados. A Seção 5 descreve o mecanismo EsPAT proposto neste trabalho. A Seção 6 apresenta um estudo de avaliação de desempenho considerando a utilização dos mecanismos para potencialização de agregação de tráfego EsPAT e Spectrum Reservation for each Node Pair (SRNP) [Zhang et al. 2015]. Por fim, a Seção 7]apresenta as conclusões e trabalhos futuros.

\section{Trabalhos relacionados}

O estabelecimento de um circuito em uma rede óptica elástica exige a reserva de um transmissor no nó de origem, um receptor no nó de destino, e uma porção do espectro óptico que consiste em um agrupamento de slots de espectro. Os slots alocados para um circuito devem ser os mesmos em cada enlace da rota. Isto é conhecido como restrição de continuidade de espectro. Além disto, os slots precisam ser consecutivos o que é conhecido como restrição de contiguidade de espectro [Chatterjee et al. 2015].

Visando a melhor utilização dos recursos da rede óptica, trabalhos da literatura apontam para o atendimento de múltiplas requisições com um mesmo circuito. Esta técnica é conhecida como agregação de tráfego [Zhang et al. 2015, Costa and Drummond 2016, Wu et al. 2017].

Alguns trabalhos consideram também a utilização de mais de um circuito para atender um cliente. Nesses casos, os dados passam por uma ou mais conversões ÓpticoEletro-Óptica nos nós intermediários [Zhang et al. 2015, Costa and Drummond 2016]. Neste trabalho esta técnica será referenciada como agregação de tráfego multihop.

Os BVTs e BV-WXCs permitem ampliar a capacidade de um circuito óptico para atender novas demandas. Para isto, são requeridos slots de espectro livres adjacentes ao canal óptico em questão. Caso contrário, o circuito óptico não pode ser expandido. Em [Zhang et al. 2013] foi proposto o mecanismo Spectrum Reservation (SR) que cria circuitos ópticos com largura de banda excedente visando potencializar a agregação de tráfego em um instante posterior.

Em [Zhang et al. 2015] o SR foi aperfeiçoado originando duas estratégias de reserva de espectro: o Spectrum Reservation for each Light Path (SRLP) e o Spectrum Reservation for each Node Pair (SRNP). O trabalho comparou os mecanismos utilizando diferentes políticas de agregação de tráfego e constatou que o SRNP apresenta melhor desempenho em todos os cenários avaliados.

A literatura apresenta diversas propostas de soluções para RMLSA e Agregação de tráfego. Segundo o nosso conhecimento, com exceção das estratégias SR, SRLP e SRNP, não existem outras propostas de mecanismos voltados para a potencialização da agregação de tráfego. Este trabalho propõe um novo mecanismo chamado Espaçamento Pró Agregação de Tráfego (EsPAT). O EsPAT interfere na tomada de decisões da solução RMLSA com o objetivo de viabilizar a expansão futura dos circuito ópticos, potencializando a agregação de tráfego.

O espaçamento entre as faixas de espectro dos circuitos ópticos provocado pelo 
EsPAT tende a reduzir as interferências entre circuitos. Isto impacta positivamente a qualidade do sinal óptico dos circuitos estabelecidos. O modelo de camada física considerado nos experimentos deste trabalho está de acordo com [Fontinele et al. 2017] e é detalhado na Seção 4.1 .

\section{Agregação de tráfego e RMLSA}

A agregação de tráfego consiste na utilização de um circuito óptico para atender a diferentes requisições na rede. A literatura apresenta duas formas de fazer agregação de tráfego: i) agregação eletrônica [Wu et al. 2017] e ii) agregação óptica [Zhang et al. 2015, Costa and Drummond 2016]. A agregação eletrônica exige a utilização de um switch para fazer a comutação dos dados de diferentes clientes e a multiplexação dos BVTs. Por outro lado, a agregação óptica exige a utilização de BVTs mais sofisticados, capazes de transmitirem simultaneamente os dados de diferentes clientes em porções do espectro óptico distintas sem separação por banda de guarda. Além disso, na agregação óptica, os BVWXCs também precisam ser mais sofisticados para poderem fazer a separação dos dados de diferentes clientes no domínio óptico.

Os BVTs com capacidade para agregação óptica são referenciados na literatura como Sliceable Bandwidth-Variable Transponder (SBVT) [Zhang et al. 2015]. O SBVT é capaz de fazer a agregação de fluxos de dados distintos no domínio óptico sem banda de guarda entre eles. Apesar dos visíveis benefícios da agregação óptica, a literatura é escassa em propostas de modelos de SBVTs bem como a modelagem das imperfeições de camada física e consumo energético destes dispositivos. A agregação óptica também exige a utilização de BV-WXCs capazes de fazer a separação dos fluxos de dados no domínio óptico.

Neste trabalho serão considerados BVTs e BV-WXCs menos sofisticados, sem capacidade para agregação óptica. Desta forma, a rede óptica elástica estudada está habilitada apenas para agregação de tráfego eletrônica. Os BVTs considerados possuem a capacidade de alterar a frequência central e a amplitude espectral utilizada na transmissão. Desta forma, os circuitos ópticos podem ser estabelecidos e ajustados de acordo com a demanda na rede.

Segundo nosso conhecimento, ainda não existe modelagem de efeitos de camada física para BVTs e BV-WXCs que fazem agregação óptica. Levando isto em consideração, este trabalho considera modelos de BVTs e BV-WXCs que permitem apenas agregação de tráfego eletrônica.

Neste trabalho é utilizada a agregação de tráfego multihop, ou seja, um circuito pode ser utilizado para levar os dados de uma requisição até um nó intermediário, a partir daí os dados são levados por outro circuito e assim sucessivamente até o nó de destino. Em cada nó intermediário há uma conversão Óptico-Eletro-Óptica (OEO) dos dados. A Figura 1 exemplifica como uma requisição entre os nós 1 e 4 pode ser atendida considerando a agregação de tráfego multihop.

A escolha da solução de agregação de tráfego aplicada a cada requisição na rede é determinada pela política de agregação de tráfego. Neste trabalho foram consideradas as políticas de agregação de tráfego Minimal Virtual Hops (MVH), Minimal Physical Hops (MPH) [Zhang et al. 2015] e Minimal Spectrum Utilization (MSU) [Wu et al. 2014]. Vale 


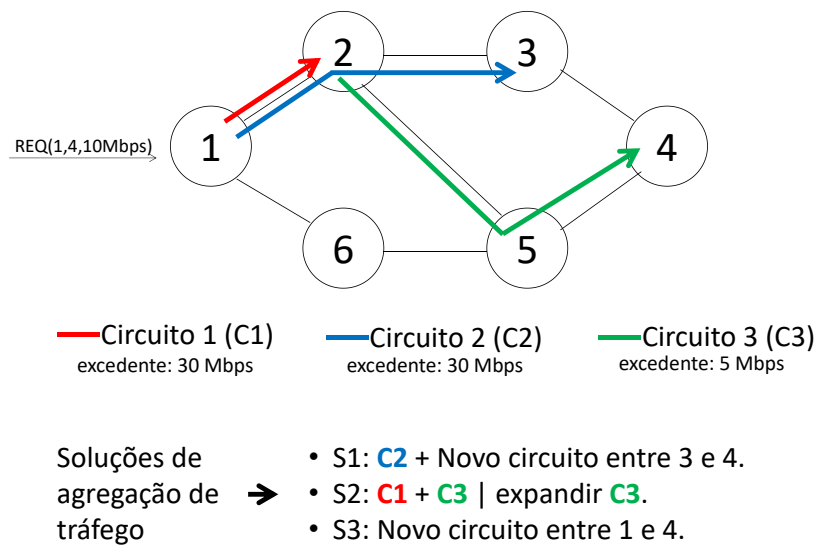

Figura 1. Possíveis soluções de agregação de tráfego para uma requisição entre os nós 1 e 4.

destacar que o mecanismo proposto neste trabalho obteve sucesso em potencializar a agregação de tráfego considerando todas as políticas de agregação de tráfego avaliadas.

O algoritmo de agregação de tráfego define de acordo com a política de agregação de tráfego adotada quais circuitos ativos na rede serão utilizados para atender à uma nova requisição. Além disso, também são determinados quais circuitos ativos serão expandidos e quais novos circuitos serão criados.

Quando há a necessidade de criar um novo circuito, os algoritmos RMLSA são acionados. Estes algoritmos são responsáveis por determinar a rota, o formato de modulação e os slots de espectro óptico que serão utilizados. Neste processo devem ser respeitadas as restrições de continuidade e contiguidade de espectro [Wang et al. 2012], a disponibilidade de BVTs e a qualidade do sinal óptico compatível com o formato de modulação escolhido [Fontinele et al. 2017].

\section{Considerações sobre os modelos de camada física, consumo de energia, BVTs e BV-WXCs}

\subsection{Modelagem de camada física}

O sinal óptico ao ser transmitido por enlaces e dispositivos ópticos perde a sua qualidade devido aos efeitos de camada física. Quando os efeitos de camada física são independentes da potência do sinal e afetam cada circuito separadamente eles são chamados de efeitos lineares. Já quando os efeitos de camada física são fortemente dependentes da potência acumulada e da potência individual dos circuitos ópticos transportados em paralelo na mesma fibra, causando interferências nos circuitos individualmente, eles são chamados de efeitos não lineares [Saradhi and Subramaniam 2009].

O efeito linear que contribui significativamente para limitar o alcance de transmissão do sinal óptico é a emissão espontânea amplificada (Amplified Spontaneous Emission - ASE) gerada nos amplificadores ópticos. E os principais efeitos não lineares são AutoModulação de Fase (Self-Phase Modulation - SPM), Modulação de Fase Cruzada (CrossPhase Modulation - XPM) e Mistura de Quadro Ondas (Four-Wave Mixing - FWM) [Beyranvand and Salehi 2013]. 
Os efeitos de camada física podem impactar significativamente na qualidade do sinal óptico tornando-a inaceitável no receptor [Beyranvand and Salehi 2013]. A relação sinal ruído (Signal to Noise Ratio - SNR) é adotada neste trabalho para mensurar a Quality of Transmission (QoT) dos circuitos ópticos. Detalhamentos sobre o cálculo de SNR adotado neste trabalho podem ser encontrados em [Fontinele et al. 2017].

\subsection{Modelagem de consumo energético}

O modelo de consumo de energia adotado [Vizcaíno et al. 2014, Ju et al. 2016] considera a contribuição dos BVTs, BV-WXCs e EDFAs. O consumo de energia de um BVT pode ser expresso como uma função da sua taxa de transmissão como mostra a Equação 1. O consumo de energia é expresso em Watts (W) na Equação 1, em que TR (Gbps) corresponde à taxa de transmissão do transponder e $s$ é a quantidade de slots de frequência utilizados. 91,333 W corresponde a um consumo de potência adicional realizada pelos BVTs.

$$
P C_{B V T}(W)=s \cdot T R \cdot 1,683+91,333
$$

O consumo de energia de um BV-WXC é expresso como na Equação 2, em que $n$ corresponde ao grau do nó e $a$ corresponde ao número de circuitos que o $\mathrm{BV}$-WXC pode adicionar ou remover. $150 \mathrm{~W}$ corresponde a um consumo de potência adicional por nó.

$$
P C_{B V-W X C}(W)=n \cdot 85+a \cdot 100+150
$$

O consumo de energia de um amplificador é considerado como $100 \mathrm{~W}$ por direção. O consumo de energia dos amplificadores ao longo de um enlace com a distância $d$ e a distância de span de amplificação $L$ é calculado pela Equação 3 . Neste trabalho, foi considerado o valor de $100 \mathrm{~km}$ para $L$ em todos os experimentos.

$$
P C_{A M P s}(W)=(\lceil d / L-1\rceil+2) \cdot 100 .
$$

\section{Espaçamento Pró Agregação de Tráfego}

A expansão de determinados circuitos, ou seja, a ampliação da faixa de espectro utilizada por eles, pode viabilizar o atendimento de novas requisições através da agregação de tráfego. Isto pode ser feito utilizando a capacidade dos BVTs e BV-WXCs de alterarem a frequência central e a largura de espectro utilizadas na transmissão, recepção e comutação óptica.

A expansão da capacidade de um circuito ativo exige a disponibilidade de slots de espectro adjacentes ao circuito em questão visando respeitar a restrição de contiguidade de espectro. Neste trabalho propomos o mecanismo de Espaçamento Pró Agregação de Tráfego (EsPAT). O EsPAT visa o espaçamento entre as faixas de espectro alocadas para cada circuito.

A Figura 2 apresenta a distribuição de espectro óptico para os circuitos $\mathrm{C} 1, \mathrm{C} 2, \mathrm{C} 3$ e C4 de duas maneiras. Na Figura 2(a) o espectro está alocado de forma mais condensada, já na Figura 2(b), o espectro foi dividido de forma mais espaçada entre os circuitos. Da forma como o espectro óptico foi alocado na Figura 2(a), os circuitos C3 e C4 não podem ser expandidos pois não há slots de espectro livres adjacentes à eles. Por outro lado, na Figura 2(b), há a possibilidade de expansão para todos os circuitos representados. 


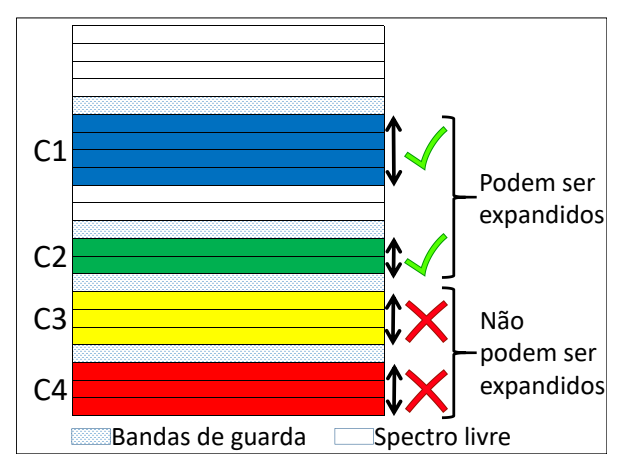

(a) Sem Espaçamento

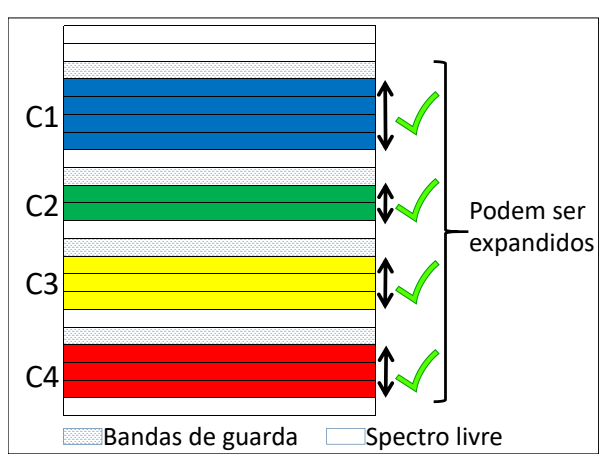

(b) Com espaçamento

Figura 2. Distribuição do espectro óptico entre circuitos.

O EsPAT utiliza o parâmetro $\sigma$ para determinar o quão espaçado deve ficar cada novo circuito em relação aos circuitos ativos na rede. Valores menores de $\sigma$ preservam as características do algoritmo de alocação de espectro, valores maiores de $\sigma$ proporcionam maior espaçamento entre os canais ópticos. O Algoritmo 1 demonstra como o EsPAT age no momento da criação de um novo circuito.

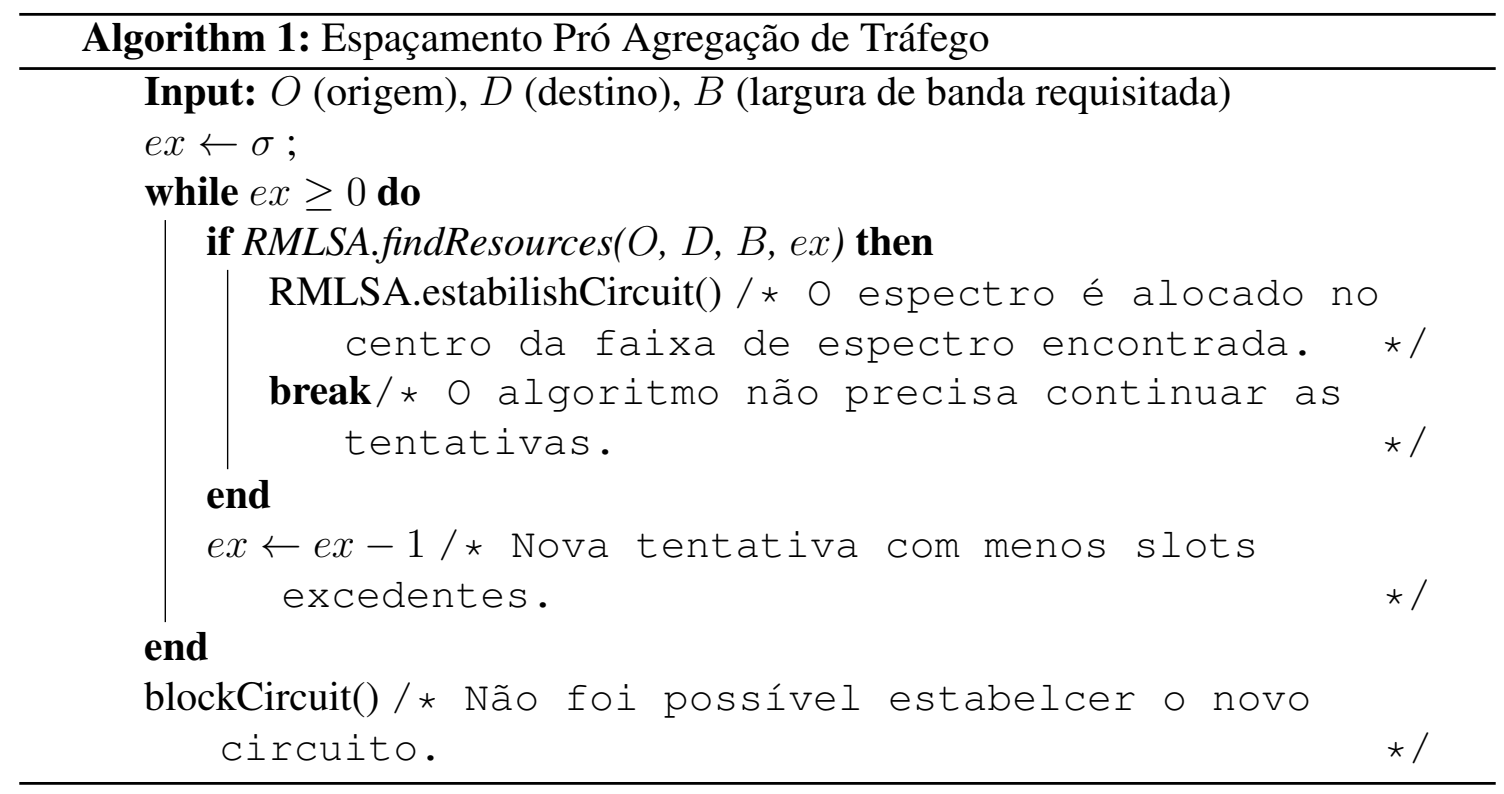

Para a implementação do EsPAT, os algoritmos RMLSA devem ser adaptados para buscarem os recursos que atendam a uma nova requisição com uma quantidade parametrizada de slots de espectro excedentes adjacentes (método findResources $(O, D, B$, ex) no Algoritmo 11). Apesar disto, o espectro alocado para o circuito deve ser suficiente apenas para atender à demanda representada por $B$.

Caso não seja encontrada uma faixa de espectro livre que atenda à demanda da nova requisição e a quantidade de slots de espectro excedentes determinada por $\sigma$, o EsPAT reduz a quantidade pretendida de slots excedentes sucessivamente até que os algoritmos RMLSA possam encontrar uma solução compatível.

$\mathrm{O}$ valor de $\sigma$ deve ser determinado durante a fase de planejamento da rede. Valores 
baixos não irão provocar espaçamento suficiente para impactar a capacidade de expansão dos circuitos e consequentemente a agregação de tráfego. Por outro lado, valores altos de $\sigma$ podem provocar a fragmentação do espectro óptico dificultando o estabelecimento de novos circuitos.

\section{Avaliação de Desempenho}

A ferramenta Slice Network Simulator (SNetS) [Fontinele et al. 2017] foi utilizada para a realização de todos os experimentos neste trabalho. Nos experimentos realizados, foi considerado um espectro óptico dividido em 400 slots de 12, 5GHz. Cada circuito tem 1 slot de espectro utilizado para banda de guarda. Os formatos de modulação utilizados foram: BPSK, QPSK, 8QAM, 16QAM, 32QAM e 64QAM.

Foram utilizadas as políticas de agregação de tráfego MPH, MVH e MSU [Zhang et al. 2015]. A solução CompleteSharing [Wang and Mukherjee 2014] foi utilizada para roteamento e alocação de espectro. Por fim, o algoritmo Modulation by QoT [Beyranvand and Salehi 2013] foi utilizado para a escolha dos formatos de modulação utilizados em cada novo circuito óptico.

Para cada cenário simulado foram feitas 10 replicações. Em cada replicação foram feitas 100.000 observações de requisições distribuídas uniformemente entre cada par de nós da rede. As larguras de banda requisitadas foram de 10, 40, 80, 100, 160, 200 e 400Gbps, com as proporções 7:6:5:4:3:2:1, respectivamente. Todos os resultados apresentam barras de erro considerando um nível de confiança de $95 \%$.

Todos os experimentos foram realizados nas topologias NSFNet e EON ilustradas na Figura 3. Por questão de espaço, serão apresentados apenas os resultados obtidos na topologia NSFNet, entretanto, todos os demais resultados estão acessíveis no repositório https://github.com/iallengabio/EsPAT. No respositório também estão disponíveis os dados brutos obtidos nas simulações, os arquivos de configuração e a ferramenta SNetS para a replicação dos experimentos.

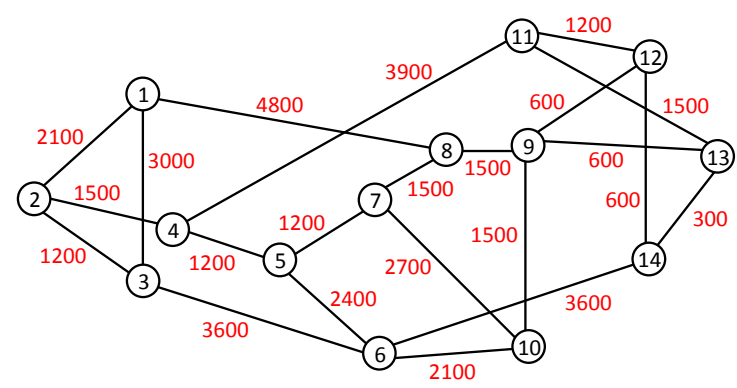

(a) NSFNet

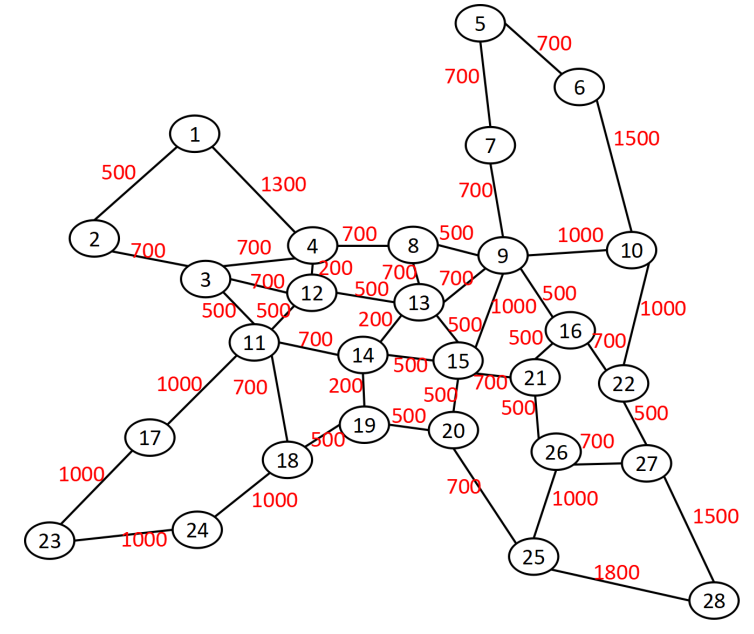

(b) EON

Figura 3. Topologias de rede utilizadas na avaliação de desempenho.

Em [Zhang et al. 2015] os autores consideram apenas um formato de modulação, 
neste cenário a reserva de slots de espectro é diretamente proporcional à reserva de largura de banda em cada canal óptico. O mecanismo SRNP proposto em [Zhang et al. 2015] foi adaptado para o cenário com múltiplos formatos de modulação e sem agregação óptica. A alteração consiste em fazer reserva de largura de banda nos circuitos estabelecidos no lugar de slots de espectro.

Durante o estabelecimento de um novo circuito, o SRNP almeja alcançar uma quantidade excedente de largura de banda não comprometida para cada par de nós na rede. Neste trabalho esta quantidade será referenciada como meta de reserva de largura de banda (MRLB).

A métrica de Bloqueio de banda (BB) representa a proporção entre a quantidade de bits que não foram transferidos devido ao bloqueio de requisições e o número total de bits que seriam transferidos caso todas as requisições fossem atendidas. A média de requisições atendidas por circuito (MRC) é a relação entre requisições atendidas e circuitos ativos. As Figuras 4 e 5 apresentam o BB e a MRC obtidos com o SRNP na topologia NSFNet com a política de agregação de tráfego MPH.

No geral, observa-se uma menor probabilidade de bloqueio com MRLB 0Gbps. Entretanto, na maioria dos casos existe sobreposição de intervalos de confiança, desta forma não há indícios suficientes para afirmar que uma solução seja significativamente mais eficiente que outra. Vale destacar que o SRNP com MRLB 0Gbps corresponde à não utilização do mecanismo.

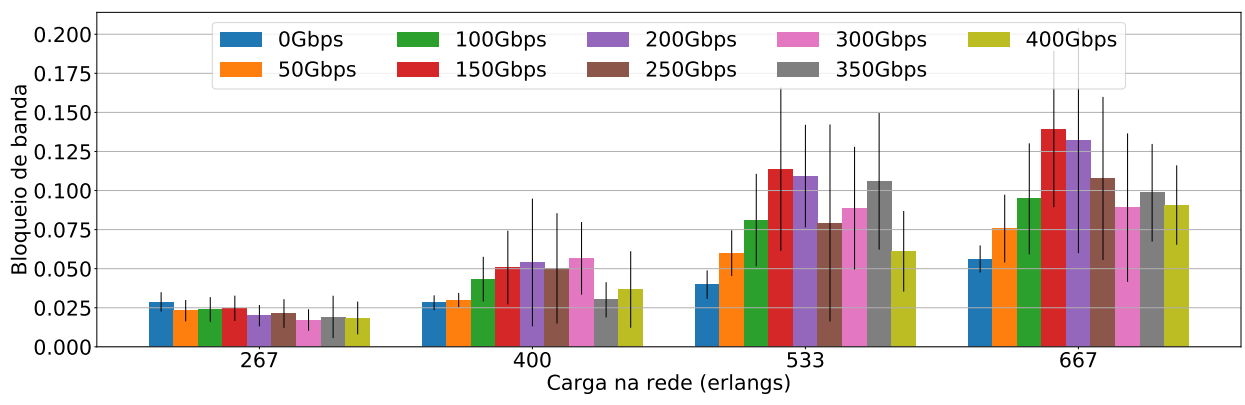

Figura 4. Bloqueio de banda obtido com a utilização do SRNP com diferentes MRLBs.

Observa-se na Figura 5 que a utilização do SRNP potencializou a agregação de tráfego uma vez que a quantidade de requisições atendidas por circuito é maior. Além disso, existe uma tendência indicando que, quanto maior for a MRLB, maior será a MRC.

As Figuras 6 6 e 7 apresentam as métricas de BB e MRC obtidas com o mecanismo EsPAT na topologia NSFNet com a política de agregação de tráfego MPH. O EsPAT com $\sigma=0$ corresponde à não utilização do mecanismo.

A Figura 6 evidencia a eficácia do mecanismo EsPAT uma vez que, a utilização do mecanismo reduz o BB em no mínimo 31,5\%, chegando até 90,3\%. nos pontos de carga 667 e 267 erlangs, respectivamente. Para valores altos de $\sigma$ o desempenho foi prejudicado, atingindo BB maior que o obtido sem a utilização do EsPAT ( $\sigma=0)$. Isto pode ser observado para $\sigma=80$ e $\sigma=90$ com carga 533 erlangs e para $\sigma=60,70,80,90$ com carga 667 erlangs. 


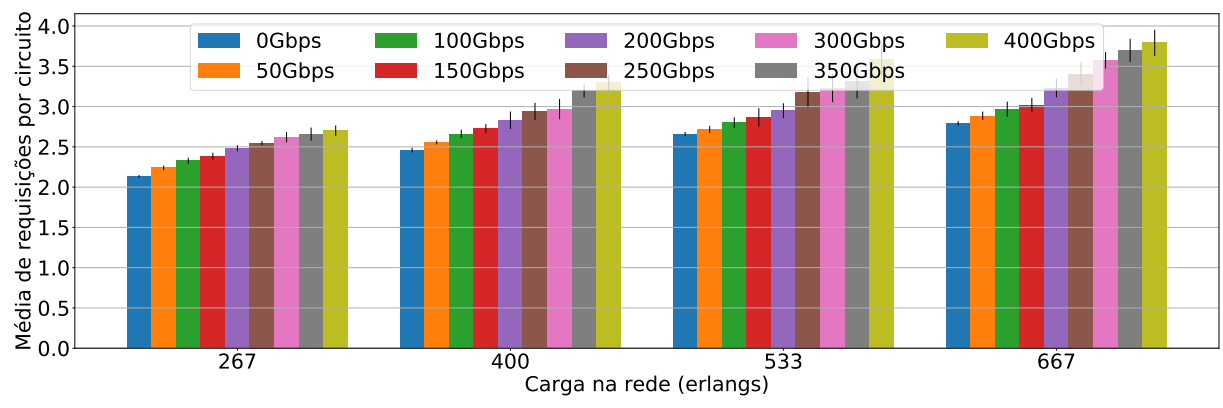

Figura 5. MRC obtida com a utilização do SRNP com diferentes MRLBs.

Ainda na Figura 6, é importante destacar que o valor ideal de $\sigma$ depende entre outras coisas da carga submetida à rede. Isto fica claro ao observar o desempenho obtido com $\sigma=60$, sob carga de 267 erlangs, o ganho em relação à não utilização do EsPAT é 92,8\%. Entretanto, sob a carga de 667 erlangs, o uso do EsPAT com $\sigma=60$ resulta em BB 10,4\% maior comparado à não utilização de nenhum mecanismo.

Durante a fase de planejamento deve ser realizado um estudo para a identificação do valor ideal para o $\sigma$. No cenário apresentado na Figura 6, o valor ideal de $\sigma$ é 40. Para este valor o EsPAT apresentou ganho de no mínimo 31,5\% e até 90,3\%.

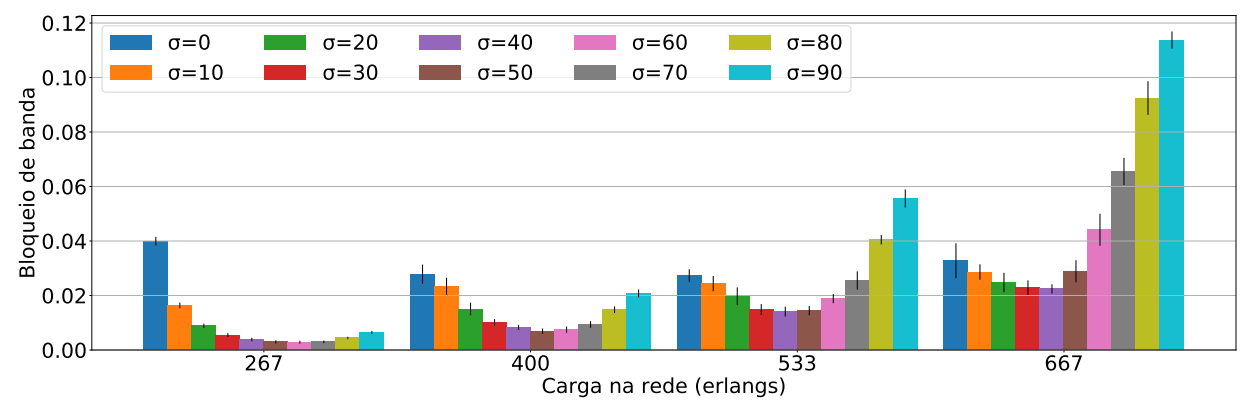

Figura 6. Bloqueio de banda obtido com a utilização do EsPAT com diferentes valores de $\sigma$

A Figura 7 apresenta a MRC obtida com a utilização do EsPAT com diferentes valores de $\sigma$. O EsPAT não faz reserva de espectro, a utilização de valores altos de $\sigma$ (acima de 60 no exemplo da Figura 7) provoca a fragmentação do espectro. Isto dificulta a expansão dos circuitos ativos e resulta em menor MRC. A utilização do EsPAT com $\sigma=40$ obteve MRC pelo menos 20\% (533 erlangs), chegando a 59,4\% (267 erlangs) maior quando comparado à não utilização do mecanismo.

Ainda sobre as Figuras 5 e 7, no geral o SRNP obteve maior MRC comparado ao EsPAT, no entanto, como o EsPAT não faz reserva de recursos, mais circuitos foram estabelecidos resultando em menor BB.

As Figuras 4, 5, 6, e 7 permitem a análise da influência da MRLB e do $\sigma$ no comportamento dos mecanismos SRNP e EsPAT. Nos demais cenários estudados os comportamentos são semelhantes. Desta forma, para otimizar o espaço serão apresentados 


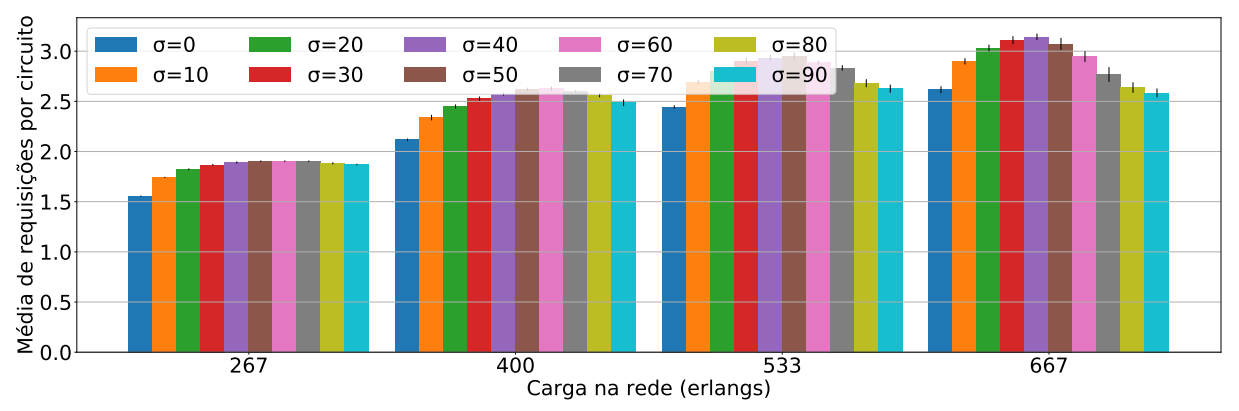

Figura 7. MRC com a utilização do EsPAT com diferentes valores de $\sigma$.

os desempenhos apenas do EsPAT com $\sigma$ ideal para cada cenário e SRNP com MRLB 200Gbps.

A Figura 8 apresenta os BBs obtidos na topologia NSFNet utilizando as políticas de agregação de tráfego (MPH, MVH e MSU). Sob carga de 267 erlangs, o SRNP apresenta redução do $\mathrm{BB}$ de $50 \%, 29,4 \%$ e $26,5 \%$ comparado à não utilização do mecanismo com as políticas MPH, MVH e MSU, respectivamente. Sob as demais cargas, a não utilização de nenhum mecanismo apresenta menor BB em relação à utilização do SRNP. Em todas as cargas e políticas de agregação de tráfego o EsPAT obteve os menores BBs. No pior caso o EsPAT apresentou redução de 16,7\%. O maior ganho obtido com a utilização do EsPAT foi de 90,3\% com a política de agregação de tráfego MPH e carga 267 erlangs.

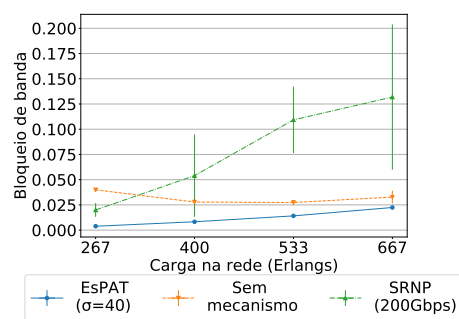

(a) MPH

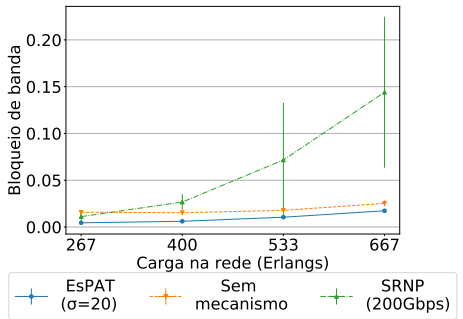

(b) $\mathrm{MVH}$

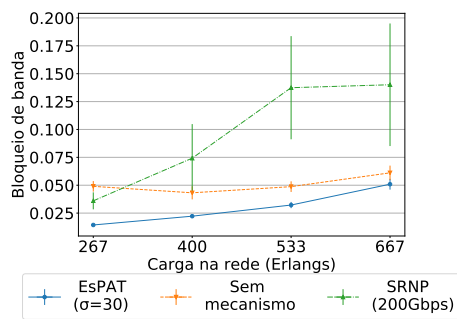

(c) MSU

Figura 8. Bloqueio de banda obtido na topologia NSFNet utilizando as política de agregação de tráfego MPH, MVH e MSU.

A Figura 9 apresenta a MRC na topologia NSFNet utilizando as políticas de agregação de tráfego MPH, MVH e MSU. Com as políticas MPH e MVH, o SRNP propicia a maior MRC. Já na MSU, o EsPAT obteve MRC superior ao SRNP. A utilização de ambos mecanismos proporciona o aumento da MRC, isto sinaliza o cumprimento do objetivo dos mecanismos de potencializar a agregação de tráfego.

A agregação de tráfego reduz a quantidade de BVTs utilizados simultâneamente na rede. Esta redução reflete tanto no consumo energético quanto na quantidade de BVTs necessários para implantar a rede. Neste sentido, os mecanismo de potencialização da agregação de tráfego promovem a redução de custos na rede óptica elástica.

As Figuras 10 e 11 apresentam a energia consumida na rede (EC) e a utilização média de BVTs (UMB) respectivamente. A Figura 10 mostra que a utilização do EsPAT 


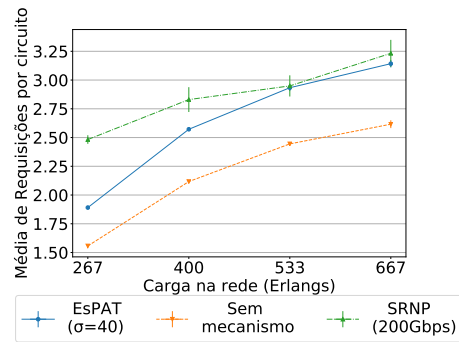

(a) $\mathrm{MPH}$

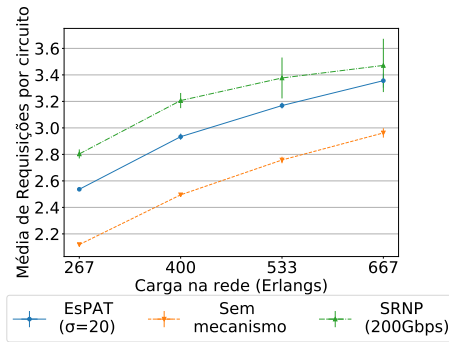

(b) $\mathrm{MVH}$

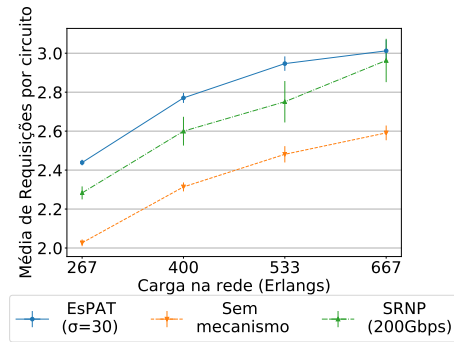

(c) MSU

Figura 9. MRC obtida na topologia NSFNet utilizando as política de agregação de tráfego MPH, MVH e MSU.

não comprometeu o consumo energético mesmo com a redução do BB (Figura 8). Apesar da formação de canais ópticos com mais slots de espectro, o que gera um maior consumo energético, o EsPAT reduziu a UMB conforme observado na Figura 11. Desta forma, o EsPAT não impactou significativamente em todos os cenários estudados.

Visando atingir a MRLB, o SRNP pode induzir o algoritmo RMLSA a criar circuitos com mais saltos físicos, desta forma, a economia energética proporcionada pela redução na UMB é compensada pelo aumento da energia consumida por amplificadores e BV-WXCs. Isto pode ser observado nas Figuras 10(b), 11(b), 10(c) e 11(c), O comportamento observado nas Figuras 10(a) e 11(a) é o inverso. Isto ocorre pois a política MPH favorece a utilização de soluções de agregação de tráfego com menor quantidade de saltos físicos, evitando a utilização de uma maior quantidade de amplificadores e BV-WXCs para atender a MRLB.

Nos experimentos realizados neste trabalho foram considerados 40 BVTs em cada nó da rede. Esta quantidade de BVTs foi dimensionada de forma a impedir bloqueios por falta de BVTs. Entretanto, de acordo com os resultados apresentados na Figura 11, o EsPAT reduz a utilização média de BVTs na rede. Na prática, o EsPAT viabiliza a redução da quantidade de BVTs por nó. Isto reduz o custo de implantação da rede e o consumo energético dos BV-WXCs segundo o modelo de consumo energético adotado.

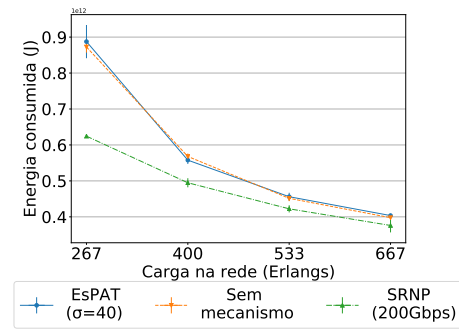

(a) $\mathrm{MPH}$

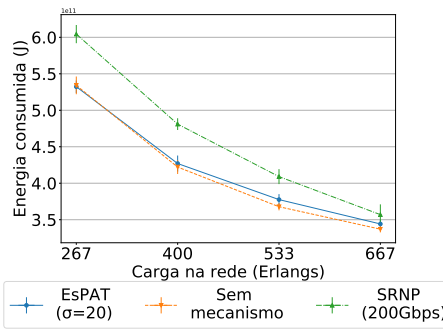

(b) $\mathrm{MVH}$

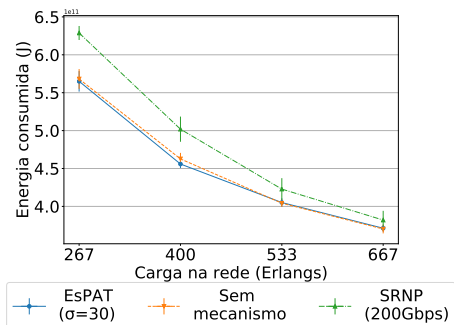

(c) MSU

Figura 10. Energia Consumida observada considerando 100.000 requisições.

\section{Conclusão}

A agregação de tráfego permite a utilização dos recursos das redes ópticas elásticas de forma mais eficiente através da redução da UMB e do desperdício de espectro óptico com 


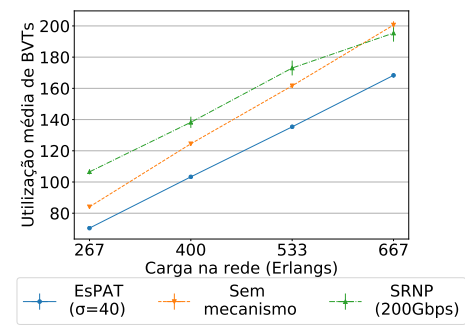

(a) $\mathrm{MPH}$

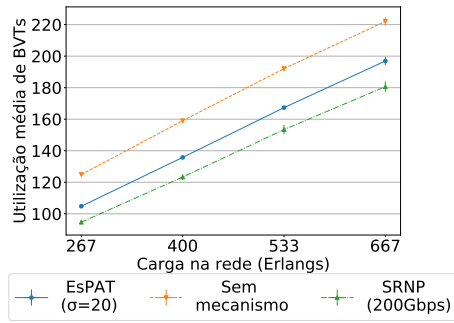

(b) $\mathrm{MVH}$

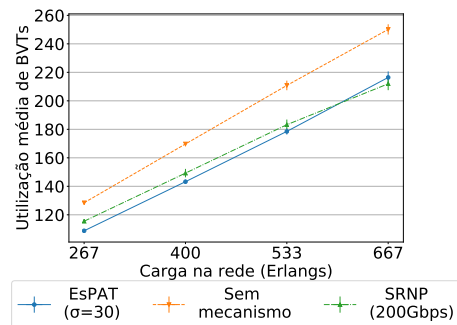

(c) MSU

Figura 11. Utilização média de BVTs na rede.

bandas de guarda. A literatura está repleta de propostas de soluções para agregação de tráfego e RMLSA. No entanto, existem poucas soluções de mecanismos independentes capazes de potencializar a agregação de tráfego.

Segundo nosso conhecimento, além do mecanismo EsPAT proposto neste trabalho, exitem apenas os mecanismos SR, SRLP e SRNP propostos em [Zhang et al. 2013] e [Zhang et al. 2015] para potencialização da agregação de tráfego. Entre os mecanismos de reserva de espectro propostos, os autores elegeram o SRNP como alternativa mais eficiente dentre as demais. Apesar disto, os experimentos realizados neste trabalho mostram que o SRNP não apresenta desempenho satisfatório considerando as redes ópticas elásticas sem agregação óptica.

O SRNP e o EsPAT foram avaliados considerando as políticas de agregação de tráfego MPH, MVH e MSU. Em todos os cenários avaliados o EsPAT apresentou o melhor desempenho em termos de bloqueio de banda com ganho de no mínimo 16,7\% e até 90,3\%. Além disso, o número médio de requisições por circuito com a utilização do EsPAT foi até $21,4 \%$ maior comparado à não utilização de nenhum mecanismo. Mesmo com o ganho de desempenho em termos de bloqueio de banda, foi mostrado que o EsPAT não comprometeu o consumo energético e ainda reduziu a UMB em pelo menos 11,3\%.

Os dados obtidos a partir dos experimentos nos permitem concluir que a adoção do mecanismo de espaçamento é mais adequada do que a reserva de espectro para as redes ópticas elásticas sem agregação óptica. Em trabalhos futuros pretendemos ajustar o $\sigma$ dinamicamente de acordo com o estado da rede e investigar o desempenho do EsPAT em redes ópticas elásticas com agregação óptica.

\section{Referências}

[Beyranvand and Salehi 2013] Beyranvand, H. and Salehi, J. A. (2013). A quality-oftransmission aware dynamic routing and spectrum assignment scheme for future elastic optical networks. Journal of Lightwave Technology, 31(18):3043-3054.

[Chatterjee et al. 2015] Chatterjee, B. C., Sarma, N., and Oki, E. (2015). Routing and spectrum allocation in elastic optical networks: A tutorial. IEEE Communications Surveys Tutorials, 17(3):1776-1800.

[Christodoulopoulos et al. 2011] Christodoulopoulos, K., Tomkos, I., and Varvarigos, E. A. (2011). Elastic bandwidth allocation in flexible ofdm-based optical networks. Journal of Lightwave Technology, 29(9):1354-1366. 
[Costa and Drummond 2016] Costa, L. and Drummond, A. (2016). Novo algoritmo RMLSA com rotemanto multihop em redes Ópticas elásticas. SBRC 2016.

[Fontinele et al. 2017] Fontinele, A., Santos, I., Neto, J. N., Campelo, D. R., and Soares, A. (2017). An efficient IA-RMLSA algorithm for transparent elastic optical networks. Computer Networks, 118(Supplement C):1 - 14.

[Ju et al. 2016] Ju, M., Zhou, F., Xiao, S., and Zhu, Z. (2016). Power-efficient protection with directed $p$-cycles for asymmetric traffic in elastic optical networks. Journal of Lightwave Technology, 34(17):4053-4065.

[Saradhi and Subramaniam 2009] Saradhi, C. V. and Subramaniam, S. (2009). Physical layer impairment aware routing (PLIAR) in WDM optical networks: Issues and challenges. Commun. Surveys Tuts., 11(4):109-130.

[Vizcaíno et al. 2014] Vizcaíno, J. L., Ye, Y., López, V., Jiménez, F., Musumeci, F., Tornatore, M., Pattavina, A., and Krummrich, P. M. (2014). Protection in optical transport networks with fixed and flexible grid: Cost and energy efficiency evaluation. Optical Switching and Networking, 11:55 - 71 .

[Wang and Mukherjee 2014] Wang, R. and Mukherjee, B. (2014). Spectrum management in heterogeneous bandwidth optical networks. Optical Switching and Networking, 11(Part A):83 - 91.

[Wang et al. 2012] Wang, Y., Cao, X., Hu, Q., and Pan, Y. (2012). Towards elastic and fine-granular bandwidth allocation in spectrum-sliced optical networks. Optical Communications and Networking, IEEE/OSA Journal of, 4(11):906-917.

[Wu et al. 2017] Wu, J., Ning, Z., and Guo, L. (2017). Energy-efficient survivable grooming in software-defined elastic optical networks. IEEE Access, 5:6454-6463.

[Wu et al. 2014] Wu, Y., Hou, W., Guo, L., Liu, Y., and Sun, Z. (2014). Green grooming in elastic optical networks. Conference on Optical Fiber Communication, Technical Digest Series.

[Zhang et al. 2015] Zhang, J., Ji, Y., Song, M., Zhao, Y., Yu, X., Zhang, J., and Mukherjee, B. (2015). Dynamic traffic grooming in sliceable bandwidth-variable transponderenabled elastic optical networks. Journal of Lightwave Technology, 33(1):183-191.

[Zhang et al. 2013] Zhang, S., Martel, C., and Mukherjee, B. (2013). Dynamic traffic grooming in elastic optical networks. IEEE Journal on Selected Areas in Communications, 31(1):4-12. 